\title{
Mandibular arteriovenous malformation
}

INSERM

\section{Source}

INSERM. (1999). Orphanet: an online rare disease and orphan drug data base. Mandibular arteriovenous malformation. ORPHA:141174

Mandibular arteriovenous malformation is a rare vascular anomaly characterized by an abnormal connection of the arterial and venous vasculature, without capillary connections, in the mandibular area, commonly presenting with minor gingival bleeding, dental loosening, lower lip numbness, facial deformity and malocclusion. This usually high-flow vascular malformation may also present with potentially life-threatening, spontaneous, or tooth extraction-induced, hemorrhagic shock. 